\title{
ESPÉCIES DE FORMIGAS QUE INTERAGEM COM AS SEMENTES DE Mabea fistulifera Mart. (EUPHORBIACEAE) ${ }^{1}$
}

\author{
Ethel Fernandes de Oliveira Peternelli² , Terezinha Maria Castro Della Lucia² e Sebastião Venâncio Martins ${ }^{3}$
}

\begin{abstract}
RESUMO - As formigas, quando atraídas por um apêndice nutritivo, produzido na semente de certas plantas, podem exercer o papel de agente predador ou dispersor das sementes. No processo de dispersão, grande número desses insetos pode interagir com sementes de determinada planta. O objetivo deste trabalho foi identificar as espécies de formigas em contato com sementes de Mabea fistulifera Mart. - uma espécie arbórea e colonizadora em áreas antrópicas no Brasil - e o tipo de interação desses insetos com as sementes, bem como determinar as espécies dispersoras. Foram realizadas coletas manuais de formigas em fragmentos de vegetação com alta densidade de $M$. fistulifera, no município de Viçosa, MG, no momento de sua visitação às sementes. As formigas capturadas foram triadas e identificadas por espécie. Além disso, durante as coletas foram feitas observações quanto ao tipo de comportamento das formigas que se associaram às sementes e ao cálculo da taxa de remoção destas, verificando-se que 16 espécies tiveram contato com estas. Acromyrmex subterraneus subterraneus, Atta sexdens rubropilosa, Ectatomma edentatum, Pachycondyla sp.1 e Pheidole sp. 2 foram, de fato, dispersoras, já que transportaram efetivamente as sementes. Ac. subterraneus subterraneus, Camponotus rufipes, Ectatomma permagnum, Megalomyrmex sp.1, Pachycondyla sp. 1, Pachycondyla sp. 2, Pheidole sp. 4, Pheidole sp. 5 e Pogonomyrmex sp. são, pela primeira vez, relatadas interagindo com sementes. A taxa de remoção das sementes de $M$. fistulifera pelas formigas foi de 85 a $97 \%$.
\end{abstract}

Palavras-chave: interação formiga-planta, mirmecocoria e dispersão de semente.

\section{INTERACTION BETWEEN ANT SPECIES AND SEEDS OF Mabea fistulifera Mart. (EUPHORBIACEAE)}

\begin{abstract}
Ants, when attracted by nutritious corpuscles produced by seeds of certain plant species, can act as predators or dispersing agents. During seed dispersal, a great number of ant species can interact with seeds of a particular plant species. The purpose of this study was to identify the ant species that interact with seeds of Mabea fistulifera Mart., a pioneer tree species in anthropic disturbed areas in Brazil. Another objective of this work was to determine the type of ant-seed interaction and the possible ant species dispersing this Euphorbiaceae. Insect hand collecting was performed in four sites where M. fistulifera is abundant in the region of Viçosa, MG, at the moment of seed visiting. The ants were taken to the laboratory and species identified. During collection, observations on ant behavior when interacting with seeds were recorded. Seed removal rate was also obtained. Sixteen species of ants interacted with the seeds; Acromyrmex subterraneus subterraneus, Atta sexdens rubropilosa, Ectatomma edentatum, Pachycondyla sp. 1 and Pheidole sp. 2 were in fact dispersing agents as they effectively transported the seeds. Ac. subterraneus subterraneus, Camponotus rufipes, Ectatomma permagnum, Megalomyrmex sp.1, Pachycondyla sp.1, Pachycondyla sp.2, Pheidole sp.4, Pheidole sp. 5 and Pogonomyrmex sp. are reported for the first time as interacting with seeds. Seed removal rate ranged from 85 to $97 \%$.
\end{abstract}

Key words: ant-plant interaction, myrmecochory and seed dispersal

\footnotetext{
${ }^{1}$ Recebido para publicação em 05.11.2003 e aceito para publicação em 10.8.2004.

${ }^{2}$ Departamento de Biologia Animal da Universidade Federal de Viçosa.

${ }^{3}$ Departamento de Engenharia Florestal da Universidade Federal de Viçosa.
} 


\section{INTRODUÇÃO}

A mirmecocoria é a dispersão de sementes por formigas (BEATTIE e CULVER, 1982). Esse fenômeno não é considerado especializado, já que há o envolvimento de uma grande diversidade de formigas associadas às sementes (GORB e BORB, 1995; PASSOS e FERREIRA, 1996). Alguns autores chegaram a essa conclusão, como Passos e Ferreira (1996), que, estudando a dispersão de sementes de Croton priscus Croizat (Euphorbiaceae), identificaram 11 espécies de formigas que interagem com as sementes. Já Culver e Beattie (1978) encontraram 12 espécies de formigas em associação com sementes do gênero Viola (Violaceae). Também Horvitz e Schemske (1986) observaram cinco espécies de formigas em contato com sementes de Calathea ovandensis Matuda (Marantaceae).

Muitos gêneros de Formicidae são relatados como dispersores de sementes, como é o caso de Formica, Myrmica e Aphaenogaster, comuns nas florestas temperadas da Europa e América do Norte, além de espécies de Rhytidoponera, Pheidole e Iridomyrmex, provenientes da Austrália (HANDEL e BEATTIE, 1990). Já nas regiões de Cerrado do Brasil foram identificados, por Leal e Oliveira (1998), vários gêneros de Attini, dispersores de sementes, como Cyphomyrmex, Mycetarotes, Mycocepurus, Myrmicocrypta, Sericomyrmex e Trachymyrmex, incluindo ainda as cortadeiras Atta e Acromyrmex. Estes últimos dois não só removeram o elaiosoma das sementes como também as transportaram a distâncias de cerca de $12 \mathrm{~m}$. Segundo esses autores, os Attini podem ter efeito benéfico na biologia das sementes de plantas superiores quando agem como dispersores secundários em florestas neotropicais.

As espécies de formigas que interagem com sementes diferem na morfologia e na biologia, assim como as espécies de plantas mirmecocóricas diferem no tamanho das suas sementes e de seus elaiosomas (GORB e GORB, 1999; GORB et al., 2000). Sementes pequenas tendem a ser removidas por formigas pequenas e sementes grandes, por formigas grandes, que possuem fortes mandíbulas. Essa relação foi estudada por Gorb e Gorb (1995), quando avaliaram a taxa de remoção de sementes de cinco espécies de plantas mirmecocóricas por Formica polyctena. Eles mostraram, ainda, que o sucesso da mirmecocoria depende da atratividade do elaiosoma (seu valor energético é o

R. Árvore, Viçosa-MG, v.28, n.5, p.733-738, 2004 principal sinal para as formigas) (GORB e GORB, 1995), do grau de polimorfismo das operárias que forrageiam (CULVER e BEATTIE, 1978; KASPARI, 1996) e de suas estratégias de forrageamento (BEATTIE e LYONS, 1975; MOREHEAD e FEENER-Jr., 1998). É importante mencionar outro aspecto relevante desse processo, que é a maneira como as formigas abocanham as sementes. A forma e o tamanho das formigas determinam a maneira pela qual elas carregam as sementes. Para isso, a formiga seleciona uma parte da semente que seja fácil de apreender com as suas mandíbulas; normalmente sementes grandes são apreendidas pelo elaiosoma e as menores o são pelo corpo da semente (GORB e GORB, 1999).

Vulgarmente conhecida como canudo-de-pito, Mabea fistulifera Mart. é uma árvore pioneira pertencente à família Euphorbiaceae (LORENZI, 1992). Seu fruto é uma tricoca, subgloboso, levemente trisulcado, de aproximadamente 15,5 a 19,0 mm de comprimento e também de diâmetro, com pilosidade acastanhada e o epimesocarpo trincado por ocasião da deiscência; a coca é bilvalvar e monospérmica. Durante o desenvolvimento dos frutos, as glândulas nectaríferas do receptáculo aumentam de duas a três vezes de tamanho e são constantemente visitadas por formigas (VIEIRA, 1991).

O papel exercido por M. fistulifera no ecossistema é relevante. Diversos animais, cerca de 67 espécies, com diferentes características morfológicas, fisiológicas e comportamentais, visitam suas flores e frutos e utilizam seus abundantes recursos florais como alimento durante a estação seca, período em que outros itens alimentares podem ser escassos (VIEIRA, 1991).

É sabido que plantas colonizadoras apresentam, geralmente, sementes pequenas, que germinam rapidamente quando dispersadas em ambientes abertos, podendo, no entanto, permanecer dormentes no solo por anos ou décadas em locais de vegetação densa (KOZLOWSKI, 1972). A ocorrência de sauveiros dentro dessas áreas abertas poderia representar uma interação formiga-planta importante no processo de dispersão secundária das sementes e no fornecimento de um ambiente propício para o desenvolvimento dessa espécie (PINTO, 1998).

Neste trabalho, os objetivos foram: i) identificar as espécies de formigas em contato com as semen- 
tes, ii) identificar o tipo de interação desses insetos com as sementes e iii) determinar as espécies dispersoras.

\section{MATERIAL E MÉTODOS}

A metodologia para os trabalhos em campo foi adaptada e modificada de Passos e Ferreira (1996).

\section{1. Época e local de coletas de formigas}

Os experimentos foram conduzidos em fragmentos vegetais no município de Viçosa, MG $\left(20^{\circ} 45^{\prime} \mathrm{S}\right.$ e $\left.42^{\circ} 55^{\prime} \mathrm{W}\right)$. Foram utilizados quatro locais, onde era grande a abundância de $M$. fistulifera, e cada local recebeu o nome de sítio: o sítio I, no Condomínio Acamari; sítio II, no Barbado - UFV; sítio III, no Apiário - UFV e sítio IV, no Belvedere - UFV. Esses experimentos foram efetuados no período de setembro a dezembro de 2001 e 2002. As formigas coletadas em campo foram levadas ao laboratório, onde foram feitas as triagens e identificação em nível de espécies, com o auxílio de chaves taxonômicas de Bolton (1994) e do mirmecólogo taxonomista Dr. Jacques Delabie, do Laboratório de Mirmecologia da CEPLAC (Itabuna, BA).

\subsection{Taxa de remoção de sementes}

Para determinar a taxa de remoção das sementes, 20 sementes frescas de $M$. fistulifera foram marcadas com tinta e colocadas em cinco pontos ao longo da área de estudo (totalizando $\mathrm{N}=100$ observações por sítio de avaliação). As sementes foram espalhadas em parcela de $0,5 \mathrm{~m}^{2}$ no chão. Após 24 horas, as sementes remanescentes nas parcelas foram contadas.

\subsection{Interação semente-formiga}

A interação de espécies de formigas com as sementes de $M$. fistulifera foi estudada nos quatro sítios de fragmentos vegetais anteriormente mencionados (item 2.1). Em cada sítio, realizaram-se sete experimentos, em sete dias consecutivos, em que 70 sementes frescas de $M$. fistulifera eram regulamente arranjadas em uma parcela de 1 x $2 \mathrm{~m}$, distante $10 \mathrm{~m}$ da árvore com frutos. A interação foi observada entre 7 e 18 horas por, pelo menos, cinco horas consecutivas. Com base na metodologia utilizada por Passos e Ferreira (1996) foram observados os seguintes comportamentos: cortar ou mastigar o elaiosoma (c), examinar o elaiosoma (e), ignorar a semente (i), remover a semente (r) e tentar remover a semente (t). Algumas formigas, as que removeram as sementes, foram seguidas para determinar a distância que elas carregavam as sementes e qual o destino destas.

\section{RESULTADOS E DISCUSSÃO}

As sementes de M. fistulifera são diplocóricas, portanto inicialmente dispersas pela própria planta por deiscência explosiva de seus frutos e depois dispersadas pelas formigas, atraídas pelo seu elaiosoma. A distância média atingida pelas sementes de M. fistulifera após a explosão dos frutos é de cerca de 6,90 $\mathrm{m}$ (dados não publicados).

\section{1. Época, local de coletas de formigas e taxa de remoção de sementes}

Durante o período de observação e coletas das formigas em campo, observou-se que em todos os sítios ocorreram diferentes espécies em associação com as sementes.

As taxas de remoção de sementes foram relativamente elevadas, variando de 85 a 97\% (Quadro 1). Todos os sítios tiveram taxas de remoção similares ou maiores que as taxas encontradas por Passos e Ferreira (1996), que trabalharam em região tropical, e superiores aos encontrados por Culver e Beattie (1978) e Horvitz e Schemske (1986) em regiões temperadas. Comparando os resultados encontrados com os dos autores já citados, pode-se predizer que a taxa de remoção de sementes parece aumentar nas regiões neotropicais, talvez por causa da maior diversidade de formigas nessas regiões, comparadas com as temperadas.

Quadro 1-Taxa(\%) de sementes de Mabea fistulifera removidas por formigas em quatro sítios. Viçosa, MG, 07/ $10 / 2002(\mathrm{~N}=100)$

Table 1 - Proportion (\%) of Mabea fistulifera seeds removed by ants in four locations. Viçosa, MG. 10/07/2002 $(N=100)$

\begin{tabular}{lcc}
\hline Sítios & $\begin{array}{c}\text { No de Sementes } \\
\text { Não-Carregadas }\end{array}$ & $\begin{array}{c}\text { \% de Sementes } \\
\text { Removidas }\end{array}$ \\
\hline I & 15 & 85 \\
II & 9 & 91 \\
III & 3 & 97 \\
IV & 14 & 86 \\
\hline
\end{tabular}




\subsection{Interação semente-formiga}

Durante as observações diretas feitas em campo, observaram-se 16 espécies de formigas interagindo com as sementes de M. fistulifera (Quadro 2). Esse resultado veio acrescentar a outros encontrados na literatura, adicionando-se nove espécies (Ac. subterraneus subterraneus, C. rufipes, Ectatomma permagnum, Megalomyrmex sp. 1, Pachycondyla sp., Pachycondyla sp. 1, Pheidole sp. 4, Pheidole sp. 5 e Pogonomyrmex sp.) a um grande número delas que interagem com sementes contendo elaiosoma (CULVER e BEATTIE, 1978; HORVITZ e SCHEMSKE, 1986; PASSOS e FERREIRA, 1996). Isso está em concordância, então, com a hipótese de que na dispersão de $M$. fistulifera não ocorre interação espécie-específica.

Em todos os testes realizados, assim que as sementes eram colocadas nas parcelas, passaram-se cerca de 30 minutos de observações até que as primeiras espécies de formigas surgissem, e grande era a atividade das operárias com as sementes. Seis espécies cortaram e, ou, mastigaram o elaiosoma; oito examinaram as sementes; apenas quatro as ignoraram e seis tentaram removê-las. Algumas espécies chegaram a transportar as sementes. As operárias de Atta transportaram-nas por cerca de $4 \mathrm{~m}$, em média, e as de Acromyrmex a $2 \mathrm{~m}$, em média, de distância do local onde as sementes eram encontradas, porém as operárias de Acromyrmex não tinham a facilidade de transportar as sementes como tinham as de Atta.

As formigas Pheidole sp. 2 foram agressivas e recrutavam numerosas operárias para ajudar no transporte das sementes. As demais espécies de Pheidole exploravam as sementes e recrutavam grande número de operárias, mas não removiam as sementes. Simplesmente manipularam-nas no local onde elas eram encontradas; removiam o elaiosoma em pequenos fragmentos, levando-os para seus ninhos. Já a espécie Camponotus rufipes também apresentava o comportamento agressivo, mas apenas explorava, tentava remover as sementes e depois as ignorava. Além de C. rufipes ignorar as sementes, tiveram esse comportamento as espécies Pogonomyrmex sp., Camponotus sp.1 e Pachycondyla striata (Quadro 2).

Quadro 2 - Comportamento observado em cada uma das 16 espécies de formigas interagindo com sementes de Mabea fistulifera. Viçosa, MG, set.-dez. 2001 e 2002

Table 2 - Behavior exhibited by each one of 16 ant species that interacted with Mabea fistulifera. Viçosa, MG. Set.-Dec./ 2001 e 2002

\begin{tabular}{lccc}
\hline Espécies & Comportamento & $\begin{array}{c}\text { Distância Média de } \\
\text { Remoção das Sementes (m) }\end{array}$ & Subfamília \\
\hline Acromyrmex subterraneus subterraneus & $\mathrm{r}$ & $1-2$ & Myrmicinae \\
Atta sexdens rubropilosa & $\mathrm{r}$ & $2-4$ & Myrmicinae \\
Camponotus rufipes & $\mathrm{i}, \mathrm{e}, \mathrm{t}, *$ & - & Formicinae \\
Camponotus sp. 1 & $\mathrm{i}$ & 3,30 & Formicinae \\
Ectatomma edentatum & $\mathrm{e}, \mathrm{t}, \mathrm{r}$ & - & Ponerinae \\
Ectatomma permagnum & $\mathrm{e}, \mathrm{t}$ & - & Ponerinae \\
Megalomyrmex sp.1 & $\mathrm{t}, \mathrm{e}, \mathrm{c}$ & - & Ponerinae \\
Pachycondyla sp.1 & $\mathrm{e}, \mathrm{t}$ & 0,15 & Ponerinae \\
Pachycondyla sp.2 & $\mathrm{t}, \mathrm{e}, \mathrm{r}$ & - & Ponerinae \\
Pachycondyla striata & $\mathrm{i}$ & - & Ponerinae \\
Pheidole sp.1 & $\mathrm{c}$ & 1,3 & Myrmicinae \\
Pheidole sp. 2 & $\mathrm{r}, \mathrm{c}, *$ & - & Myrmicinae \\
Pheidole sp. 3 & $\mathrm{e}, \mathrm{c}$ & - & Myrmicinae \\
Pheidole sp.4 & $\mathrm{c}$ & $\mathrm{M}$ & Myrmicinae \\
Pheidole sp.5 & $\mathrm{e}, \mathrm{c}$ & - & Myrmicinae \\
Pogonomyrmex sp. & $\mathrm{i}$ & Myrmicinae \\
\hline
\end{tabular}

Remover (r), cortar e mastigar (c), examinar (e), tentar remover (t) e ignorar (i).

* Agressivas. 
Foi observado que tanto operárias de Atta quanto de Acromyrmex não removiam as sementes quando as operárias de Pheidole sp. 2 estavam forrageando, pois estas eram bastante agressivas com qualquer outra espécie que tentasse remover alguma semente na área de seu forrageamento. $\mathrm{O}$ maior número de operárias de Atta e Acromyrmex que interagiram com as sementes foi visto no final de tarde, quando a atividade das operárias de Pheidole sp. 2 diminuía.

Quase todas as sementes removidas pelas formigas tiveram como destino final seus ninhos, onde os elaiosomas eram removidos e, depois, descartados nas proximidades do ninho ou em seus lixos. Isso já não ocorreu com as sementes manipuladas pelas espécies de E. edentatum, as quais tiveram um comportamento curioso: as operárias dessa espécie, ao encontrarem as sementes, examinavam-as e, em seguida, transportavam-nas para locais escondidos como debaixo de folhas e galhos, onde elas cortavam o elaiosoma. Nenhuma semente foi vista sendo levada para os ninhos dessa formiga, no período de observação. Talvez esse comportamento seja uma estratégia dessa espécie para proteger seu ninho contra predadores. No dia seguinte à realização das observações, era efetuada nova visita a todos os locais, quando, então, foram encontradas sementes sem o elaiosoma.

Das 16 espécies que interagiram com as sementes, oito pertenciam à subfamília Myrmicinae, seis à subfamília Ponerinae e duas à subfamília Formicinae (Quadro 2). Myrmicinae é uma subfamília diversificada que ocupa diversos "habitats", em regiões temperadas e tropicais (SUDD e FRANKS, 1987). As espécies coletoras de sementes "harvesting-ants" são consideradas predadoras de sementes, mas em algumas circunstâncias são dispersoras, ou seja, quando transportam as sementes para seus ninhos durante o percurso podem perder ou abandonar as sementes em locais em que elas poderão ter a chance de se estabelecer e germinar (HANDEL e BEATTIE, 1990). As formigas Ponerinae são, na maioria das vezes, predadoras de sementes, mas, assim como as formigas coletoras, podem ser dispersoras em circunstâncias especiais. Nos Formicinae, o gênero Camponotus é o que apresenta o maior número, com cerca de 1.000 espécies. Algumas delas podem interagir com as sementes que possuem elaiosoma, mas não são eficientes dispersoras (SUDD e FRANKS 1987). Já as espécies das tribos Attini e Pheidolini podem ser eficientes dispersoras diretas (LEAL e OLIVEIRA, 1998).

É importante mencionar que o elaiosoma tende a secar rapidamente após a abertura do fruto. Sementes com elaiosoma seco têm baixa atratividade para as formigas e, freqüentemente, não são dispersas por elas (MARK e OLESEN, 1996).

Das observações feitas, constatou-se, ainda, que algumas espécies de formigas são mais eficientes no transporte de sementes que outras. Tal variação pode ter importantes conseqüências ecológicas, por exemplo as sementes removidas podem estar livres de predação. Segundo Horvitz e Schemske (1986), a proteção contra a predação pode ser sugerida como uma importante força seletiva, favorecendo o mutualismo entre sementes e formigas.

Sementes sem o elaiosoma e plântulas de $M$. fistulifera foram frequientemente vistas nos montes de terra solta das formigas de Atta, indicando que o seu transporte para esses locais pode beneficiar a planta. Apesar de Atta e Pheidole serem consideradas pragas agrícolas (HÖDOBLLER e WILSON, 1990), os Attini e Pheidolini exercem efeitos benéficos quando dispersam as sementes de $C$. priscus (LEAL e OLIVEIRA, 1998; PASSOS e FERREIRA, 1996; KASPARI, 1996). De maneira semelhante, as de M. fistulifera também são dispersadas por essas espécies de formigas. A dispersão de sementes de $M$. fistulifera por formigas deve ser mais um fator que contribui para a agressividade dessa espécie na colonização de áreas degradadas, como áreas queimadas, barrancos, bordas de florestas secundárias etc.

\section{CONCLUSÃO}

Pode-se concluir que um total de 16 espécies de formigas interagem com sementes de $M$. fistulifera no município de Viçosa. Dessas, Acromyrmex subterraneus subterraneus, Atta sexdens rubropilosa, Ectatomma edentatum, Pachycondyla sp. 1 e Pheidole sp. 2 são mirmecocóricas. As formigas apresentam diferentes comportamentos ao interagirem com as sementes.

\section{AGRADECIMENTOS}

Os autores agradecem ao CNPq a concessão de bolsas de pesquisa; ao Dr. Jacques Delabie, a identificação dos Formicidae; e ao Sr. Manoel, e a Fabrícia e Gabriela, auxílio de campo.

R. Árvore, Viçosa-MG, v.28, n.5, p.733-738, 2004 


\section{REFERÊNCIAS BIBLIOGRÁFICAS}

BOLTON, B. Identification guide to the ant genera of the world. London: Harvard University, 1994. 222p.

BEATTIE, A. J.; CULVER, D. C. Inhumation: how ants and other invertebrates help seeds. Nature, v.1, p.297, 1982.

BEATTIE, A. J.; LYONS, N. Seed dispersal in Viola (Violaceae): adaptations and strategies. American Journal of Botany, v.62, n.7, p.714-722, 1975.

CULVER, D. C.; BEATTIE, A. J. Myrmecochory in Viola: dynamics of seed-ant interaction in some West Virginia species. Journal of Ecology, v. 66, p.53-72, 1978.

GORB, S. N.; GORB, E. V. Removal rates of seeds of five myrmecochorous plants by the ant Formica polyctena (Hymenoptera: Formicidae). Oikos, v.73, p.367-374, 1995.

GORB, S. N.; GORB, E. V. Dropping rates of elaiosome-bearing seeds during transport by ants (Formica polyctena Foerst.): Implications for distance dispersal. Acta Oecologica, v.20, n.5, p.509-518, 1999.

GORB, S. N.; GORB. E. V.; PUNTTILA, P. Effects of redispersal of seeds by ants on the vegetation pattern in a deciduous forest: a case study. Acta Oecologica, v. 21, n. 4-5, p.293-301, 2000.

HANDEL, S. N.; BEATTIE, A. J. Seed dispersal by ants. Scientific American, v. 263, n. 2, p. 76$83,1990$.

HÖLLDOBLER, B.; WILSON, E. O. The ants. Cambridge Harvard University Press, 1990. 733 p.

HORVITZ, C. C.; SCHEMSKE, D. W. Seed dispersal of a neotropical myrmecochore: variation in removal rates and dispersal distance.

Biotropica, v. 18, n.4, p.319-323, 1986.

KASPARI, M. Worker size and seed size selection by harvester ants in a Neotropical forest.

Oecologia, v.105, p.397-404, 1996.
KOZLOWSKI, T. T. Seed biology Importance, development and germination. New York: Academic Press. 1972, $416 \mathrm{p}$.

LEAL, I. R.; OLIVEIRA, P. S. Interactions between fungus-growing ants (Attini), fruits and seeds in cerrado vegetation in southeast Brazil.

Biotropica, v. 30, n.2, p.170-178, 1998.

LORENZI, H. As árvores brasileiras: manual de identificação e cultivo de plantas arbóreas nativas do Brasil. 2 ed., Nova Odessa: Plantarum. 1992. p.106.

MARK, S.; OLESEN, J. M. Importance of elaiosome size to removal of ant-dispersed seeds. Oecologia. V.107, p.95-101, 1996.

MOREHEAD, S. A.; FEENER-JR., D. H. Foraging behavior and morphology: seed selection in the harvester ant genus, Pogonomyrmex.

Oecologia, v.114, p.548-555, 1998.

PASSOS, L.; FERREIRA, S. O. Ant dispersal of Croton priscus (Euphorbiaceae) seeds in a tropical semideciduous forest in southeastern Brazil. Biotropica, v.28, n.4b, p.697-700, 1996.

PINTO, F. S. Efeitos da dispersão de sementes por animais e dos fatores edáficos sobre a germinação, crescimento e sobrevivência das plântulas de lobeira Solanum lycocarpum. 1998. 68f. Disertação ( Mestrado em Ecologia). Universidade de Brasília, Brasília,1998.

SUDD, J. H.; FRANKS, N. R. The behavioural ecology of ants. New York: Chapman \& Hall, 1987. 206p.

VIEIRA, M. F. Ecologia da polinização de Mabea fistulifera (Euphorbiaceae) na região de Viçosa - MG. 1991. 85f.

Dissertação (Mestrado em Biologia). Universidade Estadual de Campinas, Campinas, 1991. 